\title{
Transmuted new generalized Weibull distribution for lifetime modeling
}

\author{
Muhammad Shuaib Khan ${ }^{1, a}$, Robert King ${ }^{a}$, Irene Lena Hudson ${ }^{a}$ \\ ${ }^{a}$ School of Mathematical and Physical Sciences, The University of Newcastle, Australia
}

\begin{abstract}
The Weibull family of lifetime distributions play a fundamental role in reliability engineering and life testing problems. This paper investigates the potential usefulness of transmuted new generalized Weibull (TNGW) distribution for modeling lifetime data. This distribution is an important competitive model that contains twentythree lifetime distributions as special cases. We can obtain the TNGW distribution using the quadratic rank transmutation map (QRTM) technique. We derive the analytical shapes of the density and hazard functions for graphical illustrations. In addition, we explore some mathematical properties of the TNGW model including expressions for the quantile function, moments, entropies, mean deviation, Bonferroni and Lorenz curves and the moments of order statistics. The method of maximum likelihood is used to estimate the model parameters. Finally the applicability of the TNGW model is presented using nicotine in cigarettes data for illustration.
\end{abstract}

Keywords: new generalized Weibull distribution, moment estimation, entropies, order statistics, maximum likelihood estimation

\section{Introduction}

The probabilistic modeling approach is a traditional device to explain real world scenarios in many areas of research. Therefore, any probabilistic model defined on the positive real line can be considered as a lifetime model. The most popular model is the Weibull distribution that has been extensively used over the past decades to model lifetime data in reliability engineering, astronomy, medicine, psychology, botany, zoology, agriculture, fisheries and actuaries. The two-parameter Weibull distribution was introduced by Swedish physicist Waloddi Weibull in 1937 and has proved to be a versatile model with a wide range of applicability for analyzing lifetime data. The beauty of this model is the ability to provide reasonably accurate failure analysis and failure forecasts with extremely small samples, Abernethy (2000). Ever since, it has been extensively used for analyzing lifetime data. Many authors have introduced new distributions to model bathtub shaped instantaneous failure rates.

For example, Mudholkar and Srivastava (1993) proposed the exponentiated Weibull distribution to analyze failure data. Reliability analysis using an additive Weibull and extended Weibull models with bathtub-shaped failure rate functions were introduced by Xie and Lai (1996) and Xie et al. (2002). Nadarajah and Kotz (2006) introduced four exponentiated type distributions: exponentiated gamma, exponentiated Weibull, exponentiated Gumbel, and exponentiated Fréchet distributions. Sarhan and Zaindin (2009) introduced modified Weibull distribution. Cordeiro et al. (2013) introduced beta exponentiated Weibull distribution and studied various statistical properties with applications. Cordeiro

\footnotetext{
${ }^{1}$ Corresponding author: School of Mathematical and Physical Sciences, The University of Newcastle, Callaghan, NSW 2308, Australia. E-mail: Shuaib.stat@gmail.com
}

Published 30 September 2016 / journal homepage: http://csam.or.kr

(c) 2016 The Korean Statistical Society, and Korean International Statistical Society. All rights reserved. 
et al. (2014) proposed an increasing five-parameter Kumaraswamy modified Weibull distribution, decreasing bathtub shaped and unimodal hazard rate functions and various statistical properties with applications. A beta modified Weibull distribution was introduced by Silva et al. (2010), which includes seventeen distributions as special cases and the study of various structural properties with application. Recently Zaindin and Sarhan (2011) proposed a new generalized Weibull (NGW) distribution. The cumulative distribution function (cdf) of the NGW distribution is given by (for $x>0$ )

$$
G(x)=\left[1-\exp \left\{-\alpha x-\eta x^{\theta}\right\}\right]^{\phi},
$$

where $\theta, \phi>0$ are the shape parameters and $\alpha, \eta>0$ are the scale parameters. The probability density function corresponding to (1.1) is given by

$$
g(x)=\phi\left(\alpha+\eta \theta x^{\theta-1}\right) \exp \left\{-\alpha x-\eta x^{\theta}\right\}\left[1-\exp \left\{-\alpha x-\eta x^{\theta}\right\}\right]^{\phi-1} .
$$

The four-parameter NGW distribution generalizes the eleven lifetime distributions with an increasing and decreasing bathtub shaped hazard rate function.

Let $F_{1}$ and $F_{2}$ be the cdfs of two lifetime distributions with a common sample space, then we can define the pair of general rank transmutation proposed by Shaw and Buckley (2009) as $G_{R_{12}}(u)=$ $F_{2}\left(F_{1}^{-1}(u)\right)$ and $G_{R_{21}}(u)=F_{1}\left(F_{2}^{-1}(u)\right)$. The functions $G_{R_{12}}(u)$ and $G_{R_{21}}(u)$ both map the unit interval $I=[0,1]$ into itself, and are mutual inverses under suitable assumptions. Naturally, they satisfy $G_{R_{i j}}(0)=0$ and $G_{R_{i j}}(1)=1$ (for $i, j=1,2$ ). The quadratic rank transmutation map (QRTM) is defined by $G_{R_{12}}(u)=u+\lambda u(1-u),|\lambda| \leq 1$ from which it follows $F_{2}(x)=(1+\lambda) F_{1}(x)-\lambda F_{1}(x)^{2}$ and the corresponding probability density function (pdf) is $f_{2}(x)=f_{1}(x)\left\{(1+\lambda)-2 \lambda F_{1}(x)\right\}$, where $f_{1}(x)$ and $f_{2}(x)$ are the pdfs corresponding to the cdfs of $F_{1}(x)$ and $F_{2}(x)$ respectively. For more details about the QRTM approach and some general results, see Shaw and Buckley (2009), Bourguignon et al. (2016).

This class of transmuted distributions received considerable attention after the recent work of Shaw and Buckley (2009), which provided greater flexibility of its tails and can be applied in many areas of reliability studies. A distribution can be made more flexible by adding another parameter. The QRTM technique is one way to do so. It shows promise by expanding the range of available tail properties, as can be seen in improved goodness-of-fit results, even after adjusting for the additional parameter via measures such as the AIC. Aryal and Tsokos (2011) used this technique to propose the transmuted Weibull distribution as well as derived some mathematical properties with application. Recently Khan and King (2013a, 2013b) introduced the transmuted modified Weibull, transmuted generalized inverse Weibull distributions and formulated some of its properties with application. Khan and King (2014), Khan et al. (2014) studied the flexibility of transmuted inverse Weibull distributions and studied various structural properties with an application to survival data. Khan and King (2015) recently proposed the transmuted modified inverse Rayleigh distribution and formulated some of its properties with application to real data. Elbatal and Aryal (2013) proposed the transmuted additive Weibull distribution with application to reliability data. Merovci (2013) proposed the transmuted Rayleigh distribution. Tian et al. (2014) introduced and studied the transmuted linear exponential distribution with application to reliability data. Sharma et al. (2014) have also proposed a transmuted inverse Rayleigh distribution with application to survival data that represents the primary motivation for studying the transmuted new generalized Weibull (TNGW) distribution for modeling survival data and also investigate the shapes, skewness, kurtosis and tail variations using simulation. The TNGW distribution is also called the transmuted exponentiated modified Weibull distribution recently proposed by Ashour and Eltehiwy (2013). We can obtain the TNGW distribution using the QRTM technique by taking $G(x)$ to be the cdf of the NGW distribution as the baseline model. This research 
investigates the potential usefulness of the TNGW distribution by adding the transmuted parameter $\lambda$ that offers more flexibility to model lifetime data. We study several mathematical properties of the five-parameter TNGW distribution with application to nicotine in cigarettes data.

The structure of this paper is as follows. In Section 2, we present the analytical shapes of the density and hazard functions. Some statistical properties such as the quantile functions, moment estimation and moment generating function are addressed in Section 3. Entropies, mean deviation, Bonferroni and Lorenz curves are derived in Section 4. In Section 5, we derive the density function and moments of order statistics. Maximum likelihood estimates (MLEs) of the unknown parameters are discussed in Section 6. A simulation study is also conducted to examine the bias and mean square error of maximum likelihood estimators in Section 6. Section 7 addresses the potentiality of the TNGW model by means of the nicotine in cigarettes data and associated inferences, followed by concluding remarks.

\section{Transmuted new generalized Weibull distribution}

A positive random variable $X$ has TNGW distribution with parameters $\alpha, \theta, \eta, \phi>0$ and the transmuting parameter $|\lambda| \leq 1, x>0$. Using the QRTM technique, we can obtain five-parameter TNGW distribution defined by

$$
\begin{aligned}
f(x) & =\frac{\phi\left(\alpha+\eta \theta x^{\theta-1}\right) \exp \left\{-\alpha x-\eta x^{\theta}\right\} v_{2}(x)}{\left[1-\exp \left\{-\alpha x-\eta x^{\theta}\right\}\right]^{1-\phi}}, \\
v_{g}(x) & =\left\{1+\lambda-g \lambda\left[1-\exp \left\{-\alpha x-\eta x^{\theta}\right\}\right]^{\phi}\right\}, \quad g=1,2 .
\end{aligned}
$$

The cdf corresponding to (2.1) is given by

$$
F(x)=\left[1-\exp \left\{-\alpha x-\eta x^{\theta}\right\}\right]^{\phi} v_{1}(x)
$$

Here $v_{g}(x)$ is used to simplify the presentation of this density function. The parameter $\alpha$ is the location parameter, the parameter $\eta$ controls the scale of the distribution, whereas the parameters $\theta$ and $\phi$ control the shape of the distribution respectively. The parameter $\lambda$ is a kind of transmuting parameter that provides more flexibility in the TNGW model. The TNGW distribution is the extended form of the NGW distribution and reduces to the base model when the parameter $\lambda=0$. If $X$ is a random variable with density function (2.1), we write $X \sim \operatorname{TNGW}(x ; \alpha, \theta, \eta, \phi, \lambda)$. The associated reliability and hazard rate functions follow from (2.1) and (2.3) are defined by

$$
R(x)=1-\left[1-\exp \left\{-\alpha x-\eta x^{\theta}\right\}\right]^{\phi} v_{1}(x),
$$

and

$$
h(x)=\frac{\phi\left(\alpha+\eta \theta x^{\theta-1}\right) \exp \left\{-\alpha x-\eta x^{\theta}\right\} v_{2}(x)}{\left[1-\exp \left\{-\alpha x-\eta x^{\theta}\right\}\right]^{1-\phi}\left[1-\left[1-\exp \left\{-\alpha x-\eta x^{\theta}\right\}\right]^{\phi} v_{1}(x)\right]}
$$

Figure 1 illustrates the shapes of the TNGW pdf with some selected choice of parameters. When the cdf of the TNGW distribution has zero value then it represents no failure components. Figure 2 illustrates the hazard function of the TNGW distribution with a different choice of parameters. The 

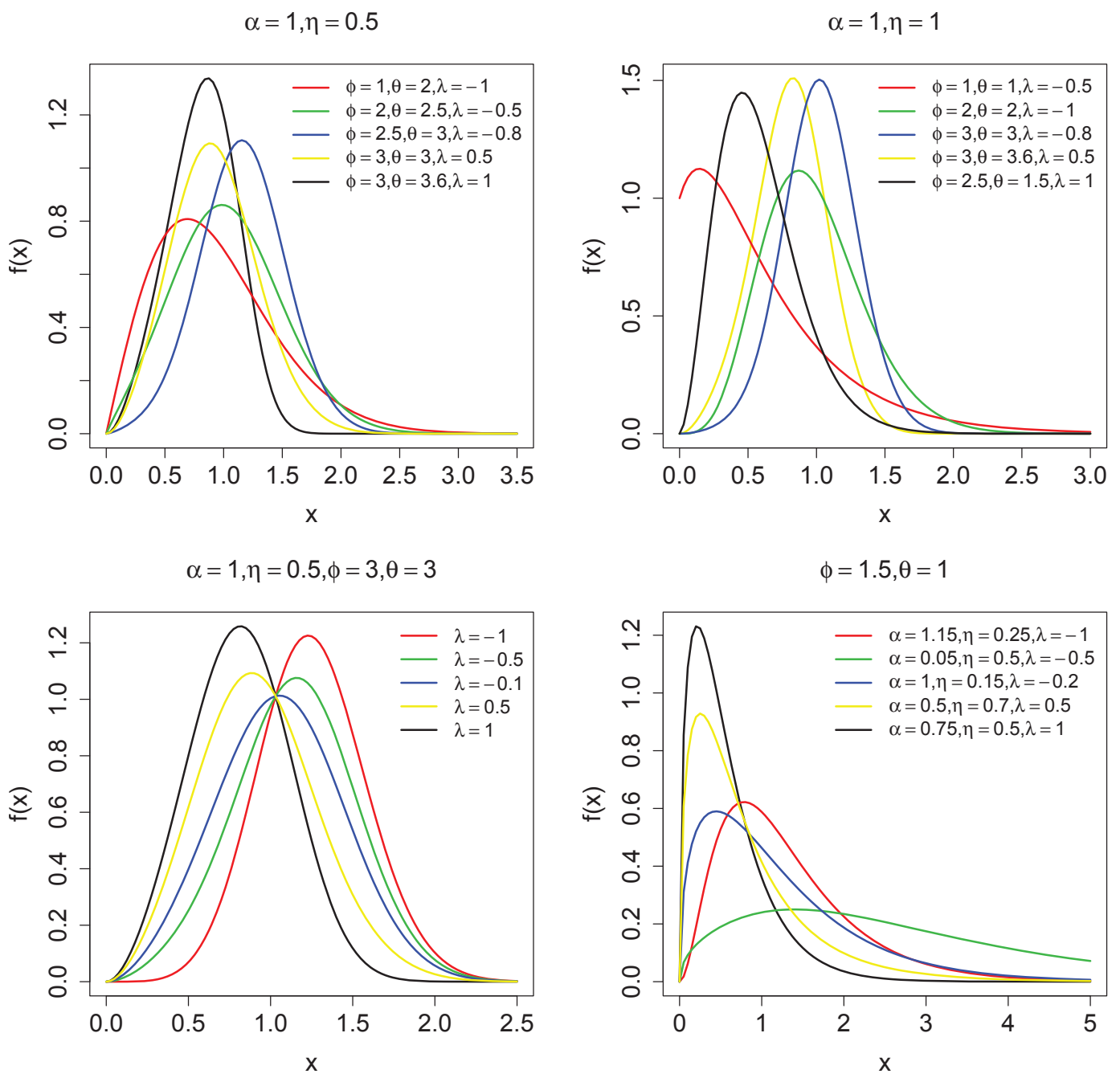

Figure 1: Plots of the TNGW pdf for some selected values of the parameters.

distribution has increasing, decreasing and constant behaviors for hazard rates. The TNGW distribution provides a wide usage of the Weibull family of lifetime distributions. The TNGW model can be used in more complex situations and provides more flexibility in real world scenarios. The TNGW distribution contains several well-known distributions as special cases several well-known distributions. Twenty-three distributions included as special cases of the TNGW distribution are displayed in Table 1.

\section{Moments and quantiles}

This section presents the quantile analysis, $k^{\text {th }}$ moment and moment generating function of the TNGW distribution. 

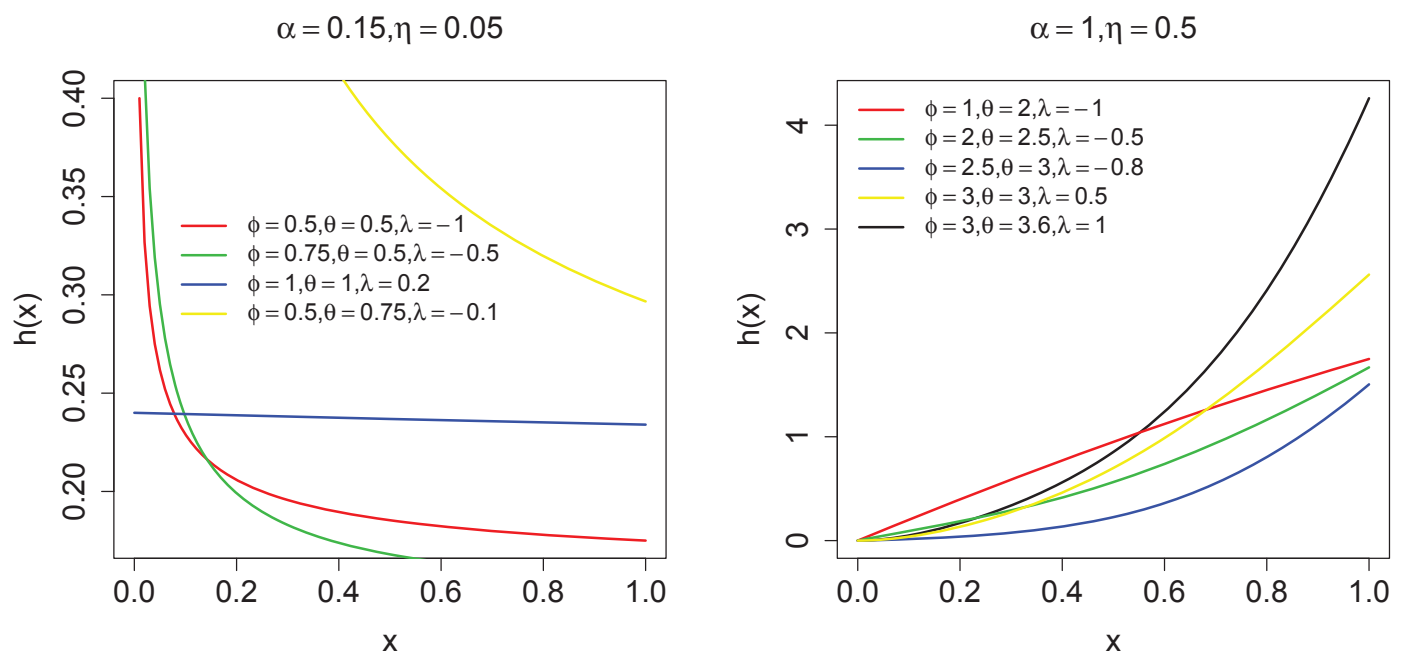

Figure 2: Plots of the TNGW hazard function for some selected values of the parameters.

Table 1: Sub-models of the transmuted NGW distribution

\begin{tabular}{|c|c|c|c|c|c|}
\hline Distribution & $\alpha$ & $\theta$ & $\eta$ & $\phi$ & $\lambda$ \\
\hline TNGE & - & 1 & - & - & - \\
\hline TNGR & - & 2 & - & - & - \\
\hline TMW & - & - & - & 1 & - \\
\hline TMR & - & 2 & - & 1 & - \\
\hline TME & - & 1 & - & 1 & - \\
\hline TGW & 0 & - & - & - & - \\
\hline TGR & 0 & 2 & - & - & - \\
\hline TGE & 0 & 1 & - & - & - \\
\hline TW & 0 & - & - & 1 & - \\
\hline TR & 0 & 2 & - & 1 & - \\
\hline $\mathrm{TE}$ & 0 & 1 & - & 1 & - \\
\hline NGW & - & - & - & - & 0 \\
\hline NGR & - & 2 & - & - & 0 \\
\hline NGE & - & 1 & - & - & 0 \\
\hline MW & - & - & - & 1 & 0 \\
\hline MR & - & 2 & - & 1 & 0 \\
\hline ME & - & 1 & - & 1 & 0 \\
\hline GW & 0 & - & - & - & 0 \\
\hline GR & 0 & 2 & - & - & 0 \\
\hline GE & 0 & 1 & - & - & 0 \\
\hline W & 0 & - & - & 1 & 0 \\
\hline $\mathrm{R}$ & 0 & 2 & - & 1 & 0 \\
\hline $\mathrm{E}$ & 0 & 1 & - & 1 & 0 \\
\hline
\end{tabular}

$\mathrm{T}$ = transmuted; $\mathrm{M}$ = modified; $\mathrm{N}$ = new; $\mathrm{G}$ = generalized; $\mathrm{W}$ = Weibull; $\mathrm{R}$ = Rayleigh; $\mathrm{E}$ = exponential.

\subsection{Quantile and median}

The quantile $x_{q}$ of the TNGW distribution is the real solution of the following equation

$$
\eta x_{q}^{\theta}+\alpha x_{q}+\ln \left\{1-\left(\frac{(1+\lambda)-\sqrt{(1+\lambda)^{2}-4 \lambda q}}{2 \lambda}\right)^{\frac{1}{\phi}}\right\}=0 .
$$


The above equation (3.1) has no closed form solution in $x_{q}$, in general the TNGW model contains twenty-three quantile models as special cases. For the non-linear structure, we can obtain these quantile models by using numerical method such as Newton-Raphson technique. Among twenty-three quantile models, we formulate the mathematical expressions for eleven quantile models for the transmuted distributions in closed form solution as special cases by substituting the parametric values in equation (3.1) as given by

$$
U(\phi, \lambda, q)=\ln \left\{1-\left(\frac{(1+\lambda)-\sqrt{(1+\lambda)^{2}-4 \lambda q}}{2 \lambda}\right)^{\frac{1}{\phi}}\right\} .
$$

1. The $q^{\text {th }}$ quantile of the $\operatorname{TNGE}(x ; \alpha, \eta, \phi, \lambda)$ by substituting $\theta=1$

$$
x_{q}=\frac{-1}{\alpha+\eta} U(\phi, \lambda, q)
$$

2. The $q^{\text {th }}$ quantile of the $\operatorname{TNGR}(x ; \alpha, \eta, \phi, \lambda)$ by substituting $\theta=2$

$$
x_{q}=\frac{-\alpha+\sqrt{\alpha^{2}-4 \eta U(\phi, \lambda, q)}}{2 \eta} .
$$

3. The $q^{\text {th }}$ quantile of the $\operatorname{TMW}(x ; \alpha, \theta, \eta, \lambda)$ by substituting $\phi=1$

$$
\eta x_{q}^{\theta}+\alpha x_{q}+U(1, \lambda, q)=0
$$

4. The $q^{\text {th }}$ quantile of the $\operatorname{TMR}(x ; \alpha, \eta, \lambda)$ by substituting $\phi=1, \theta=2$

$$
x_{q}=\frac{-\alpha+\sqrt{\alpha^{2}-4 \eta U(1, \lambda, q)}}{2 \eta} .
$$

5. The $q^{\text {th }}$ quantile of the $\operatorname{TME}(x ; \alpha, \phi, \lambda)$ by substituting $\phi=1, \theta=1$

$$
x_{q}=\frac{-1}{\alpha+\eta} U(1, \lambda, q) \text {. }
$$

6. The $q^{\text {th }}$ quantile of the $\operatorname{TGW}(x ; \theta, \eta, \phi, \lambda)$ by substituting $\alpha=0$

$$
x_{q}=\left\{\frac{-1}{\eta} U(\phi, \lambda, q)\right\}^{\frac{1}{\theta}} .
$$

7. The $q^{\text {th }}$ quantile of the $\operatorname{TGR}(x ; \eta, \phi, \lambda)$ by substituting $\alpha=0, \theta=2$

$$
x_{q}=\sqrt{\frac{-1}{\eta} U(\phi, \lambda, q)} .
$$



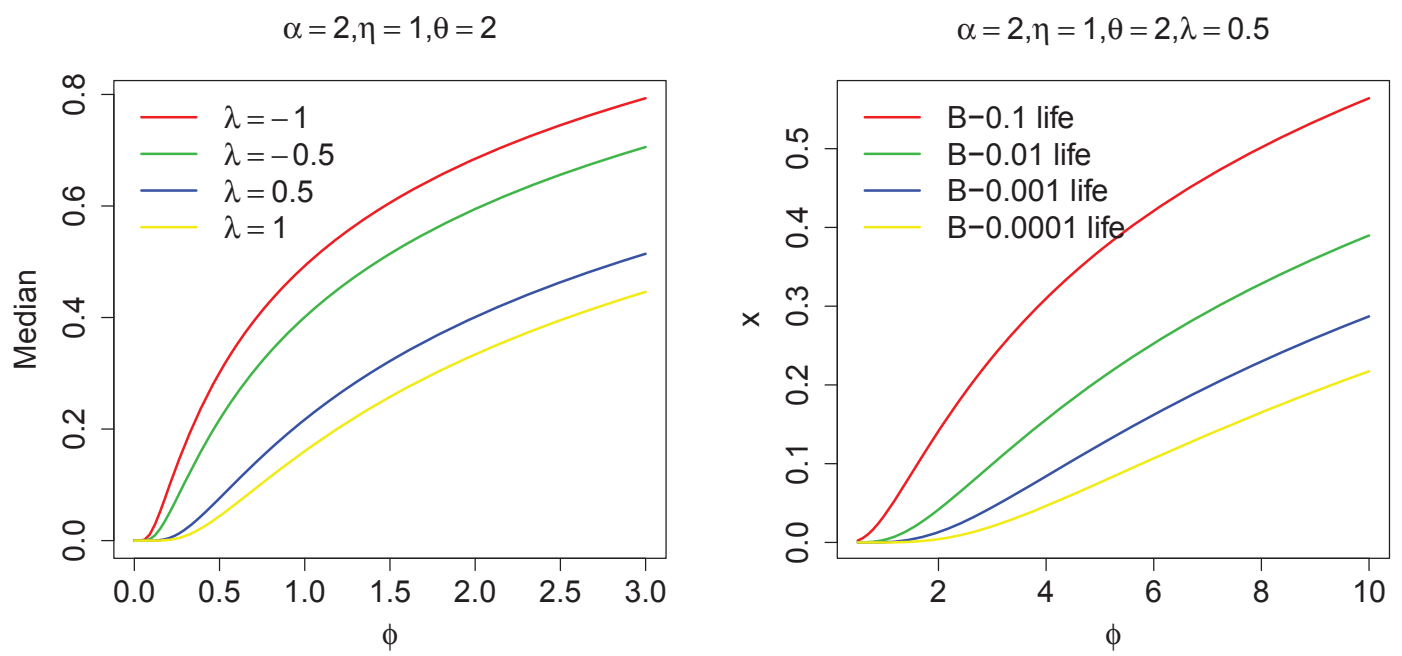

Figure 3: Median and B-life of the TNGW distribution.

8. The $q^{\text {th }}$ quantile of the $\operatorname{TGE}(x ; \eta, \phi, \lambda)$ by substituting $\alpha=0, \theta=1$

$$
x_{q}=\frac{-1}{\eta} U(\phi, \lambda, q) .
$$

9. The $q^{\text {th }}$ quantile of the $\operatorname{TW}(x ; \theta, \eta, \lambda)$ by substituting $\alpha=0, \phi=1$

$$
x_{q}=\left(\frac{U(1, \lambda, q)}{\eta}\right)^{\frac{1}{\theta}} .
$$

10. The $q^{\text {th }}$ quantile of the $\operatorname{TR}(x ; \eta, \lambda)$ by putting $\alpha=0, \phi=1, \theta=2$

$$
x_{q}=\sqrt{\frac{U(1, \lambda, q)}{\eta}} .
$$

11. The $q^{\text {th }}$ quantile of the $\operatorname{TE}(x ; \eta, \lambda)$ by putting $\alpha=0, \phi=1, \theta=1$

$$
x_{q}=\frac{U(1, \lambda, q)}{\eta} .
$$

By substituting $q=0.5$ in (3.1) we obtain the median of the TNGW distribution. Figure 3 shows the median life to illustrate the effect of transmuting parameter $\lambda$ for some selected choice of parameters. Figure 3 also shows the B-life (or percentile life) of the TNGW distribution as a function of the shape parameter $\phi$. We evaluate the performance of Bowley skewness and Moors kurtosis using the measure based on quantiles as a function of the transmuting parameter $\lambda$. Graphical representations of the Bowley skewness and Moors kurtosis show the three phases of these plots and are displayed in Figure 4, respectively. 

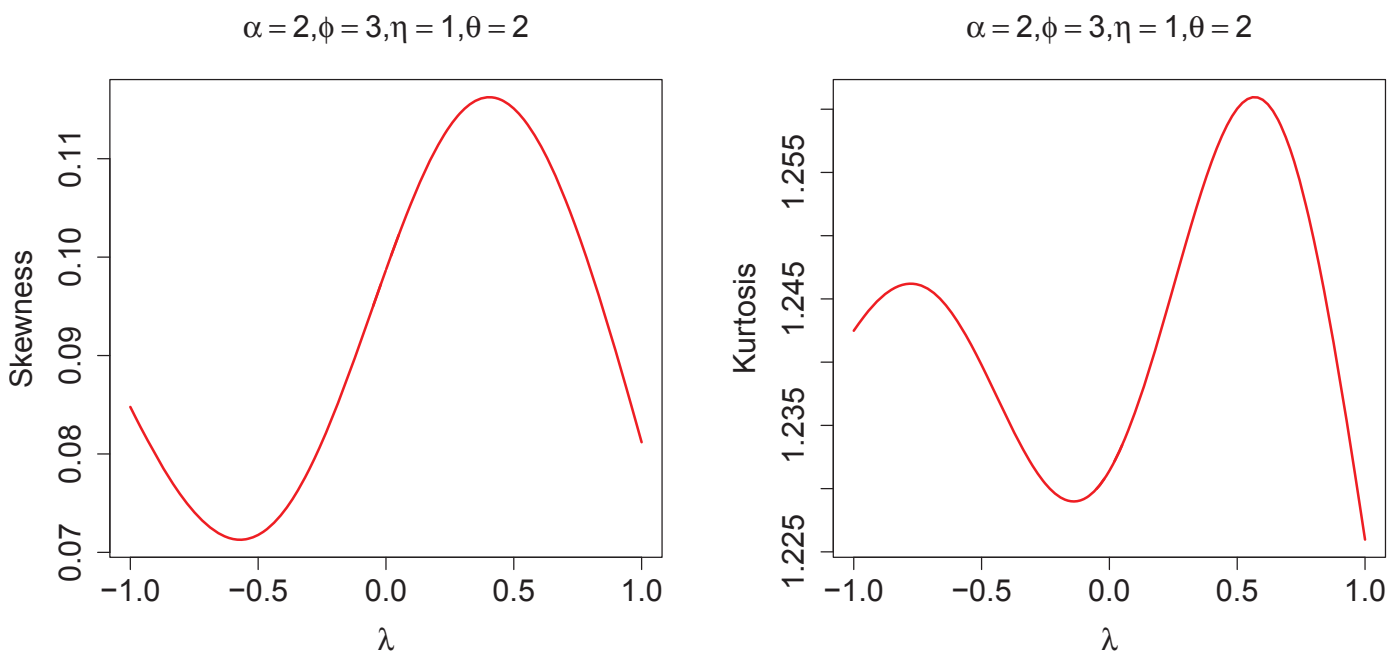

Figure 4: Skewness and kurtosis of the TNGW for different values of $\lambda$.

\subsection{Moments}

Theorem 1. If $X$ has the $T N G W(x ; \alpha, \theta, \eta, \phi, \lambda)$ with $|\lambda| \leq 1$, then the $k^{\text {th }}$ moment of $X$ is $\tilde{\mu}_{k}=(1+\lambda) \sum_{m, n=0}^{\infty}\left(\begin{array}{c}\phi-1 \\ m\end{array}\right) \frac{\eta^{n} \phi(-1)^{m+n}}{n !(m+1)^{-n}} \psi(\alpha, \theta, \eta, m, n, k)-2 \lambda \sum_{m, n=0}^{\infty}\left(\begin{array}{c}2 \phi-1 \\ m\end{array}\right) \frac{\eta^{n} \phi(-1)^{m+n}}{n !(m+1)^{-n}} \psi(\alpha, \theta, \eta, m, n, k)$, where

$$
\psi(\alpha, \theta, \eta, m, n, k)=\frac{\alpha \Gamma(k+\theta n+1)}{(\alpha(m+1))^{k+\theta n+1}}+\frac{\theta \eta \Gamma(k+\theta n+\theta)}{(\alpha(m+1))^{k+\theta n+\theta}} .
$$

Proof: The $k^{\text {th }}$ moment of the TNGW distribution is as follows

$$
\tilde{\mu}_{k}=\int_{0}^{\infty} x^{k} \frac{\phi\left(\alpha+\eta \theta x^{\theta-1}\right) \exp \left\{-\alpha x-\eta x^{\theta}\right\} v_{2}(x)}{\left[1-\exp \left\{-\alpha x-\eta x^{\theta}\right\}\right]^{1-\phi}} d x
$$

using (2.1) and (2.2) the above integral can be written as

$$
\hat{\mu}_{k}=(1+\lambda) \int_{0}^{\infty} x^{k} \frac{\phi\left(\alpha+\eta \theta x^{\theta-1}\right) \exp \left\{-\alpha x-\eta x^{\theta}\right\}}{\left[1-\exp \left\{-\alpha x-\eta x^{\theta}\right\}\right]^{1-\phi}} d x-2 \lambda \int_{0}^{\infty} x^{k} \frac{\phi\left(\alpha+\eta \theta x^{\theta-1}\right) \exp \left\{-\alpha x-\eta x^{\theta}\right\}}{\left[1-\exp \left\{-\alpha x-\eta x^{\theta}\right\}\right]^{1-2 \phi}} d x .
$$

Using the binomial expansion the above equation reduces to

$$
\begin{aligned}
\hat{\mu}_{k}= & (1+\lambda) \sum_{m=0}^{\infty}\left(\begin{array}{c}
\phi-1 \\
m
\end{array}\right) \phi \alpha(-1)^{m} \int_{0}^{\infty} x^{k} \exp \left\{-\alpha x(m+1)-\eta x^{\theta}(m+1)\right\} d x \\
& +(1+\lambda) \sum_{m=0}^{\infty}\left(\begin{array}{c}
\phi-1 \\
m
\end{array}\right) \phi \eta \theta(-1)^{m} \int_{0}^{\infty} x^{k+\theta+1} \exp \left\{-\alpha x(m+1)-\eta x^{\theta}(m+1)\right\} d x
\end{aligned}
$$


Table 2: Moments values of the TNGW distribution

\begin{tabular}{crcccc}
\hline \hline$(\alpha, \theta, \eta, \phi)$ & \multicolumn{1}{c}{$\lambda$} & $\dot{\mu}_{1}$ & $\dot{\mu}_{2}$ & $\dot{\mu}_{3}$ & $\dot{\mu}_{4}$ \\
\hline & -1.0 & 0.7365 & 1.0861 & 2.4618 & 7.6323 \\
$1,0.5,1,1$ & -0.5 & 0.5954 & 0.8382 & 1.8687 & 5.7545 \\
& 0.5 & 0.5000 & 0.5555 & 1.0000 & 2.5185 \\
& 1.0 & 0.3333 & 0.2222 & 0.2222 & 0.2963 \\
\hline \multirow{2}{*}{$1,1,1,1$} & -1.0 & 0.7500 & 0.8750 & 1.4062 & 2.9062 \\
& -0.5 & 0.6250 & 0.6875 & 1.0781 & 2.2031 \\
& 0.5 & 0.3750 & 0.3125 & 0.4218 & 0.7968 \\
$1,2,2,2$ & 1.0 & 0.2500 & 0.1250 & 0.0937 & 0.0938 \\
& -1.0 & 0.7656 & 0.6588 & 0.6263 & 0.6499 \\
& -0.5 & 0.6846 & 0.5535 & 0.5066 & 0.5129 \\
$2,3,2,3$ & 0.5 & 0.5224 & 0.3429 & 0.2671 & 0.2391 \\
& 1.0 & 0.4414 & 0.2376 & 0.1474 & 0.1022 \\
\hline \hline
\end{tabular}

Table 3: Moments based measures of the TNGW distribution

\begin{tabular}{crccccc}
\hline \hline$(\alpha, \theta, \eta, \phi)$ & \multicolumn{1}{c}{$\lambda$} & Mean & Var & CV & CS & CK \\
\hline \multirow{3}{*}{$1,0.5,1,1$} & -1.0 & 0.7365 & 0.5436 & 1.0011 & 2.1481 & 10.258 \\
& -0.5 & 0.5954 & 0.4836 & 1.1681 & 2.3592 & 11.583 \\
& 0.5 & 0.5000 & 0.3055 & 1.1054 & 2.4681 & 12.475 \\
& 1.0 & 0.3333 & 0.1111 & 1.0001 & 2.0000 & 9.0027 \\
\hline \multirow{2}{*}{$1,1,1$} & -1.0 & 0.7500 & 0.3125 & 0.7453 & 1.6096 & 7.0810 \\
& -0.5 & 0.6250 & 0.2968 & 0.8717 & 1.7144 & 7.5045 \\
& 0.5 & 0.3750 & 0.1718 & 1.1055 & 2.4658 & 12.472 \\
$1,2,2,2$ & 1.0 & 0.2500 & 0.0625 & 1.0000 & 1.9968 & 9.0256 \\
& -1.0 & 0.7656 & 0.0726 & 0.3521 & 0.5448 & 3.4351 \\
& -0.5 & 0.6846 & 0.0848 & 0.4254 & 0.4669 & 3.2145 \\
$2,3,2,3$ & 0.5 & 0.5224 & 0.0699 & 0.5065 & 0.8010 & 3.8797 \\
& 1.0 & 0.4414 & 0.0427 & 0.4685 & 0.5392 & 3.1849 \\
\hline \hline & -1.0 & 0.7331 & 0.0435 & 0.2847 & 0.2194 & 3.0218 \\
& -0.5 & 0.6650 & 0.0552 & 0.3535 & 0.1036 & 2.8772 \\
& 0.5 & 0.5288 & 0.0508 & 0.4265 & 0.4384 & 3.0592 \\
\hline
\end{tabular}

$\mathrm{CV}=$ coefficient of variation; $\mathrm{CS}=$ coefficient of skewness; $\mathrm{CK}=$ coefficient of kurtosis.

$$
\begin{aligned}
& -2 \lambda \sum_{m=0}^{\infty}\left(\begin{array}{c}
2 \phi-1 \\
m
\end{array}\right) \phi \alpha(-1)^{m} \int_{0}^{\infty} x^{k} \exp \left\{-\alpha x(m+1)-\eta x^{\theta}(m+1)\right\} d x \\
& -2 \lambda \sum_{m=0}^{\infty}\left(\begin{array}{c}
2 \phi-1 \\
m
\end{array}\right) \phi \eta \theta(-1)^{m} \int_{0}^{\infty} x^{k+\theta+1} \exp \left\{-\alpha x(m+1)-\eta x^{\theta}(m+1)\right\} d x
\end{aligned}
$$

the above integral yields the following $k^{\text {th }}$ moment,

$$
\begin{aligned}
\tilde{\mu}_{k}= & (1+\lambda) \sum_{m, n=0}^{\infty}\left(\begin{array}{c}
\phi-1 \\
m
\end{array}\right) \frac{\eta^{n} \phi(-1)^{m+n}}{n !(m+1)^{-n}} \psi(\alpha, \theta, \eta, m, n, k) \\
& -2 \lambda \sum_{m, n=0}^{\infty}\left(\begin{array}{c}
2 \phi-1 \\
m
\end{array}\right) \frac{\eta^{n} \phi(-1)^{m+n}}{n !(m+1)^{-n}} \psi(\alpha, \theta, \eta, m, n, k) .
\end{aligned}
$$


The important features and characterizations of the TNGW distribution can be studied using equation (3.3). The values of the first four ordinary moments for some selected choices of parameters are shown in Table 2. Using ordinary moments, we obtained the mean, variance, coefficient of variation, coefficient of skewness and coefficient of kurtosis that are displayed in Table 3. The results in these tables show that as the shape parameters increases as the values of skewness and kurtosis decreases. These values can be determined numerically by using R and SAS languages.

Theorem 2. If $X$ has the $T N G W(x ; \alpha, \theta, \eta, \phi, \lambda)$ with $|\lambda| \leq 1$, then the moment generating function (mgf) of $X$ is given by

$$
\begin{aligned}
M_{x}(t)= & (1+\lambda) \sum_{r=0}^{\infty} \sum_{p, q=0}^{\infty}\left(\begin{array}{c}
\phi-1 \\
p
\end{array}\right) \frac{\eta^{p} \phi t^{r}(-1)^{p+q}}{q ! r !(p+1)^{-n}} \omega(\alpha, \theta, \eta, p, q, r) \\
& -2 \lambda \sum_{r=0}^{\infty} \sum_{p, q=0}^{\infty}\left(\begin{array}{c}
2 \phi-1 \\
p
\end{array}\right) \frac{\eta^{p} \phi(-1)^{p+q}}{q ! r !(p+1)^{-n}} \omega(\alpha, \theta, \eta, p, q, r),
\end{aligned}
$$

where

$$
\omega(\alpha, \theta, \eta, p, q, r)=\frac{\alpha \Gamma(r+\theta q+1)}{(\alpha(p+1))^{r+\theta q+1}}+\frac{\theta \eta \Gamma(r+\theta q+\theta)}{(\alpha(p+1))^{r+\theta q+\theta}}
$$

Proof: By definition

$$
\begin{aligned}
M_{x}(t)= & (1+\lambda) \int_{0}^{\infty} e^{t x} \frac{\phi\left(\alpha+\eta \theta x^{\theta-1}\right) \exp \left\{-\alpha x-\eta x^{\theta}\right\}}{\left[1-\exp \left\{-\alpha x-\eta x^{\theta}\right\}\right]^{1-\phi}} d x \\
& -2 \lambda \int_{0}^{\infty} e^{t x} \frac{\phi\left(\alpha+\eta \theta x^{\theta-1}\right) \exp \left\{-\alpha x-\eta x^{\theta}\right\}}{\left[1-\exp \left\{-\alpha x-\eta x^{\theta}\right\}\right]^{1-2 \phi}} d x .
\end{aligned}
$$

Using Taylor series expansions, the above integrals reduce to

$$
\begin{aligned}
M_{x}(t)= & (1+\lambda) \sum_{r=0}^{\infty} \sum_{p=0}^{\infty}\left(\begin{array}{c}
\phi-1 \\
p
\end{array}\right) \frac{t^{r} \phi \alpha(-1)^{p}}{r !} \int_{0}^{\infty} x^{r} \exp \left\{-\alpha x(p+1)-\eta x^{\theta}(p+1)\right\} d x \\
& +(1+\lambda) \sum_{r=0}^{\infty} \sum_{p=0}^{\infty}\left(\begin{array}{c}
\phi-1 \\
m
\end{array}\right) \frac{t^{r} \phi \eta \theta(-1)^{p}}{r !} \int_{0}^{\infty} x^{r+\theta+1} \exp \left\{-\alpha x(p+1)-\eta x^{\theta}(p+1)\right\} d x \\
& -2 \lambda \sum_{r=0}^{\infty} \sum_{p=0}^{\infty}\left(\begin{array}{c}
2 \phi-1 \\
p
\end{array}\right) \frac{t^{r} \phi \alpha(-1)^{p}}{r !} \int_{0}^{\infty} x^{r} \exp \left\{-\alpha x(p+1)-\eta x^{\theta}(p+1)\right\} d x \\
& -2 \lambda \sum_{r=0}^{\infty} \sum_{p=0}^{\infty}\left(\begin{array}{c}
2 \phi-1 \\
p
\end{array}\right) \frac{t^{r} \phi \eta \theta(-1)^{p}}{r !} \int_{0}^{\infty} x^{r+\theta+1} \exp \left\{-\alpha x(p+1)-\eta x^{\theta}(p+1)\right\} d x
\end{aligned}
$$


the above integral yields the $M_{x}(t)$ as

$$
\begin{aligned}
M_{x}(t)= & (1+\lambda) \sum_{r=0}^{\infty} \sum_{p, q=0}^{\infty}\left(\begin{array}{c}
\phi-1 \\
p
\end{array}\right) \frac{\eta^{p} \phi t^{r}(-1)^{p+q}}{q ! r !(p+1)^{-n}} \omega(\alpha, \theta, \eta, p, q, r) \\
& -2 \lambda \sum_{r=0}^{\infty} \sum_{p, q=0}^{\infty}\left(\begin{array}{c}
2 \phi-1 \\
p
\end{array}\right) \frac{\eta^{p} \phi(-1)^{p+q}}{q ! r !(p+1)^{-n}} \omega(\alpha, \theta, \eta, p, q, r) .
\end{aligned}
$$

\section{Entropy and mean deviation}

The entropy of a random variable $X$ with probability density from the $\operatorname{TNGW}(x ; \alpha, \theta, \eta, \phi, \lambda)$ is a measure of variation of the uncertainty. The Rényi entropy approaches the Shannon entropy when $\rho \rightarrow 1$. The Rényi (1961) introduced the entropy denoted as, $I_{R}(\rho)$, for $X$ is a measure of variation of uncertainty and is defined as

$$
I_{R}(\rho)=\frac{1}{1-\rho} \log \left\{\int_{0}^{\infty} f(x)^{\rho} d x\right\},
$$

where $\rho>0$ and $\rho \neq 1$. The integral in $I_{R}(\rho)$ for the $T N G W(x ; \alpha, \theta, \eta, \phi, \lambda)$ can be defined by substituting (2.1) and (2.2) in (4.1) as

$$
I_{R}(\rho)=\frac{1}{1-\rho} \log \left\{\int_{0}^{\infty} \frac{\phi^{\rho}\left(\alpha+\eta \theta x^{\theta-1}\right)^{\rho} \exp \left\{-\alpha \rho x-\eta \rho x^{\theta}\right\} v_{2}(x)^{\rho}}{\left[1-\exp \left\{-\alpha x-\eta x^{\theta}\right\}\right]^{\rho(1-\phi)}} d x\right\},
$$

the above integral reduces to

$$
I_{R}(\rho)=\frac{1}{1-\rho} \log \left\{\sum_{i, j, k=0}^{\infty} z_{\phi, \rho, \lambda, i, j} \int_{0}^{\infty} x^{k(\theta-1)} \exp \left\{-\alpha x(\rho+j)-\eta x^{\theta}(\rho+j)\right\} d x\right\},
$$

where

$$
z_{\phi, \rho, \lambda, i, j}=\phi^{\rho}\left(\begin{array}{c}
\rho \\
i
\end{array}\right)\left(\begin{array}{c}
\rho \\
k
\end{array}\right)\left(\begin{array}{c}
\rho(\phi-1)+\phi i \\
j
\end{array}\right)\left(\frac{\eta \theta}{\alpha}\right)^{k}\left(\frac{2 \lambda}{1+\lambda}\right)^{i}(-1)^{i+j}(1+\lambda)^{\rho} \alpha^{\rho} .
$$

Finally, we obtain the TNGW Rényi entropy as

$$
\begin{aligned}
I_{R}(\rho)= & \frac{\rho}{1-\rho} \log \alpha+\frac{\rho}{1-\rho} \log \phi+\frac{\rho}{1-\rho} \log (1+\lambda) \\
& +\frac{1}{1-\rho} \log \left\{\sum_{i, j=0}^{\infty} \sum_{k, m=0}^{\infty}\left(\begin{array}{c}
\rho \\
i
\end{array}\right)\left(\begin{array}{c}
\rho \\
k
\end{array}\right)\left(\begin{array}{c}
\rho(\phi-1)+\phi i \\
j
\end{array}\right)\left(\frac{2 \lambda}{1+\lambda}\right)^{i} \frac{(-1)^{i+j+m}}{m !} V_{j, k, m}\right\},
\end{aligned}
$$

where

$$
V_{j, k, m}=\frac{\eta^{m}(\rho+j)^{m}}{\alpha(\rho+j)^{k(\theta-1)-\theta m+1}}\left(\frac{\eta \theta}{\alpha}\right)^{k} \Gamma(k(\theta-1)-\theta m-1) .
$$


Table 4: Rényi entropy values for the TNGW distribution

\begin{tabular}{crrrrr}
\hline \hline$(\alpha, \theta, \eta, \phi)$ & $\lambda$ & $\rho=2$ & $\rho=3$ & $\rho=4$ & $\rho=5$ \\
\hline \multirow{3}{*}{$1,1,1,1$} & -1.0 & 0.2891 & 0.2627 & 0.3698 & 0.4703 \\
& -0.5 & 0.2833 & 0.2602 & 0.3697 & 0.4741 \\
& 0.5 & 0.0828 & 0.0325 & 0.0018 & -0.0410 \\
\hline \multirow{2}{*}{$1,2,2,2$} & 1.0 & -0.3010 & -0.3635 & -0.4014 & -0.4273 \\
& -1.0 & -0.0321 & -0.0636 & -0.0827 & -0.0958 \\
& -0.5 & 0.0080 & -0.0224 & -0.0412 & -0.0541 \\
$2,3,2,3$ & 0.5 & -0.0553 & -0.0869 & -0.1062 & -0.1193 \\
& 1.0 & -0.1415 & -0.1697 & -0.1872 & -0.1993 \\
\hline & -1.0 & -0.1299 & -0.1605 & -0.1791 & -0.1918 \\
& -0.5 & -0.0745 & -0.1045 & -0.1230 & -0.1358 \\
$2,4,2,5$ & 0.5 & -0.0996 & -0.1280 & -0.1456 & -0.1578 \\
& 1.0 & -0.1732 & -0.1994 & -0.2158 & -0.2272 \\
\hline \hline
\end{tabular}

The $\beta$-or ( $q$-entropy) was introduced by Havrda and Charvát (1967), and is defined as

$$
I_{H}(\beta)=\frac{1}{\beta-1}\left\{1-\int_{0}^{\infty} f(x)^{\beta} d x\right\},
$$

where $\beta>0$ and $\beta \neq 1$. Suppose $X$ has the TNGW distribution then by substituting (2.1) and (2.2) in (4.2), we obtain

$$
I_{H}(\beta)=\frac{1}{\beta-1}\left\{1-\int_{0}^{\infty} \frac{\phi^{\beta}\left(\alpha+\eta \theta x^{\theta-1}\right)^{\beta} \exp \left\{-\alpha \beta x-\eta \beta x^{\theta}\right\} v_{2}(x)^{\beta}}{\left[1-\exp \left\{-\alpha x-\eta x^{\theta}\right\}\right]^{\beta(1-\phi)}} d x\right\}
$$

the above integral yields the TNGW $\beta$-entropy as

$$
I_{H}(\beta)=\frac{1}{\beta-1}\left\{1-\sum_{i, j=0}^{\infty} \sum_{k, m=0}^{\infty} \frac{\xi_{\phi, \beta, \lambda, i, j}(-1)^{m} \eta^{m}(\beta+j)^{m}}{m ! \alpha(\beta+j)^{k(\theta-1)-\theta m+1}} \Gamma(k(\theta-1)-\theta m-1)\right\},
$$

where

$$
\xi_{\phi, \beta, \lambda, i, j}=(\phi \alpha)^{\beta}\left(\begin{array}{c}
\beta \\
i
\end{array}\right)\left(\begin{array}{c}
\beta \\
k
\end{array}\right)\left(\begin{array}{c}
\beta(\phi-1)+\phi i \\
j
\end{array}\right)\left(\frac{\eta \theta}{\alpha}\right)^{k}\left(\frac{2 \lambda}{1+\lambda}\right)^{i}(-1)^{i+j}(1+\lambda)^{\beta} .
$$

Table 4 lists the values of Rényi entropy of the TNGW distribution for selected values of the parameters. Table 5 lists the values of $\beta$-entropy of the TNGW distribution for selected values of the parameters.

If $X$ has the TNGW distribution, then we can derive the mean deviation about the mean and about the median from the following equations

$$
\delta_{1}=\int_{0}^{\infty}|x-\mu| f(x) d x \quad \text { and } \quad \delta_{2}=\int_{0}^{\infty}|x-M| f(x) d x .
$$


Table 5: $\beta$-entropy values for the TNGW distribution

\begin{tabular}{crrrrr}
\hline \hline$(\alpha, \theta, \eta, \phi)$ & $\lambda$ & $\beta=2$ & $\beta=3$ & $\beta=4$ & $\beta=5$ \\
\hline \multirow{3}{*}{$1,1,1,1$} & -1.0 & 0.3333 & 0.2334 & 0.1809 & 0.1484 \\
& -0.5 & 0.2500 & 0.1584 & 0.1107 & 0.0809 \\
& 0.5 & -0.4166 & -0.8916 & -1.7369 & -3.4587 \\
& 1.0 & -1.0000 & -2.1666 & -5.0000 & -12.550 \\
\hline \multirow{2}{*}{$1,2,2,2$} & -1.0 & -0.0767 & -0.1703 & -0.2571 & -0.3545 \\
& -0.5 & 0.0183 & -0.0545 & -0.1096 & -0.1614 \\
& 0.5 & -0.1359 & -0.2463 & -0.3606 & -0.5000 \\
$2,3,2,3$ & 1.0 & -0.3850 & -0.5926 & -0.8816 & -1.3175 \\
& -1.0 & -0.3488 & -0.5468 & -0.8150 & -1.2134 \\
& -0.5 & -0.1872 & -0.3089 & -0.4464 & -0.6238 \\
$2,4,2,5$ & 0.5 & -0.2578 & -0.4016 & -0.5779 & -0.8188 \\
& 1.0 & -0.4899 & -0.7526 & -1.1468 & -1.7771 \\
\hline \hline & -1.0 & -0.7204 & -1.2129 & -2.0811 & -3.7062 \\
& -0.5 & -0.4680 & -0.7555 & -1.1914 & -1.9056 \\
& 0.5 & -0.4131 & -0.6487 & -0.9883 & -1.5179 \\
\hline
\end{tabular}

The mean $\mu$ is given in equation (3.3) and the median $M$ is obtained from equation (3.1). These measures are calculated using the relationships:

$$
\delta_{1}=2[\mu F(\mu)-\psi(\mu)] \text { and } \delta_{2}=\mu-2 \psi(M) .
$$

The quantity $\psi(q)$ is used to determine the Bonferroni and Lorenz curves, which are very useful in econometrics and finance, reliability and survival analysis, demography, insurance and medical sciences. For a given probability $p$, they can be constructed from $(3.1)$ and $B(P)=\psi(q) / P \mu$ and $L(P)=\psi(q) / \mu$.

$$
\begin{aligned}
\psi(q)= & (1+\lambda) \sum_{i, j=0}^{\infty}\left(\begin{array}{c}
\phi-1 \\
i
\end{array}\right) \frac{\eta^{j} \phi(-1)^{i}(i+1)^{j}}{j !(\alpha(i+1))^{\theta j+2}} U_{\alpha, \theta, \eta, i, j} \\
& -2 \lambda \sum_{i, j=0}^{\infty}\left(\begin{array}{c}
2 \phi-1 \\
i
\end{array}\right) \frac{\eta^{j} \phi(-1)^{i}(i+1)^{j}}{j !(\alpha(i+1))^{\theta j+2}} U_{\alpha, \theta, \eta, i, j}
\end{aligned}
$$

where

$$
U_{\alpha, \theta, \eta, i, j}=\alpha \gamma(\theta j+2, \alpha q(i+1))+\frac{\theta \eta}{[\alpha(i+1)]^{\theta-1}} \gamma(\theta j+\theta+1, \alpha q(i+1))
$$

\section{Order statistics}

The density of the $r^{\text {th }}$ order statistic $X_{(r)}$ of a random sample drawn from the TNGW distribution with $|\lambda| \leq 1$, with the density function of $X_{(r)}$ is given by

$$
f_{r: n}(x)=\frac{(F(x))^{r-1}(1-F(x))^{n-r} f(x)}{B(r, n-r+1)}, \quad x>0,
$$

where $B(r, n-r+1)$ is the Beta function and it is a normalizing constant, by substituting (2.1) and (2.3) in (5.1), we obtain

$$
f_{r: n}(x)=n\left(\begin{array}{c}
n-1 \\
r-1
\end{array}\right) \sum_{i=0}^{n-r}\left(\begin{array}{c}
n-r \\
i
\end{array}\right)(-1)^{i} V_{r: i}(x)
$$


where

$$
V_{r: i}(x)=\frac{\phi\left(\alpha+\eta \theta x^{\theta-1}\right) \exp \left\{-\alpha x-\eta x^{\theta}\right\} v_{1}(x)^{r+i-1} v_{2}(x)}{\left[1-\exp \left\{-\alpha x-\eta x^{\theta}\right\}\right]^{1-\phi(r+i)}} .
$$

The pdf of $r^{\text {th }}$ order statistics of the TNGW distribution reduces to the combining terms as

$$
f_{r \cdot n}(x)=n\left(\begin{array}{c}
n-1 \\
r-1
\end{array}\right) \sum_{i=0}^{n-r} \sum_{j, k=0}^{\infty}(-1)^{i+j+k} U_{i, j, k} z_{k}(x),
$$

where

$$
U_{i, j, k}=\left(\begin{array}{c}
n-r \\
i
\end{array}\right)\left(\begin{array}{c}
r+i-1 \\
j
\end{array}\right)\left(\begin{array}{c}
\phi(r+i+j)-1 \\
k
\end{array}\right)(1+\lambda)^{r+i-1}\left(\frac{\lambda}{1+\lambda}\right)^{j},
$$

and

$$
z_{k}(x)=\phi\left(\alpha+\eta \theta x^{\theta-1}\right) \exp \left\{-\alpha x(k+1)-\eta x^{\theta}(k+1)\right\} v_{2}(x) .
$$

Using (5.2), the $S^{\text {th }}$ moment of the $r^{\text {th }}$ order statistics $X_{(r)}$ is given by

$$
\mu_{s}^{n: r}=\sum_{i=0}^{n-r} \sum_{j=0}^{\infty} \psi_{i, j}\left\{(1+\lambda) \sum_{k, m=0}^{\infty} \frac{(-1)^{k+m} \eta^{m} \tau_{k, m} c_{i, j, k, 0}}{(k+1)^{-m} m !}-2 \lambda \sum_{k, m=0}^{\infty} \frac{(-1)^{k+m} \eta^{m} \tau_{k, m} c_{i, j, k, 1}}{(k+1)^{-m} m !}\right\},
$$

where $c_{i, j, k, g}=\left(\begin{array}{c}\phi(r+i+j+g)-1 \\ k\end{array}\right), g=0,1$

$$
\begin{aligned}
\psi_{i, j} & =n\left(\begin{array}{c}
n-1 \\
r-1
\end{array}\right)\left(\begin{array}{c}
n-r \\
i
\end{array}\right)\left(\begin{array}{c}
r+i-1 \\
j
\end{array}\right)(1+\lambda)^{r+i-1}\left(\frac{\lambda}{1+\lambda}\right)^{j}, \\
\tau_{k, m} & =\frac{\alpha \Gamma(S+\theta m+1)}{(\alpha(k+1))^{S+\theta m+1}}+\frac{\eta \theta \Gamma(S+\theta m+\theta)}{(\alpha(k+1))^{S+\theta m+\theta}} .
\end{aligned}
$$

\section{Maximum likelihood estimation}

Consider the random samples $x_{1}, x_{2}, \ldots, x_{n}$ consisting of $n$ observations from the TNGW distribution and $\Theta=(\alpha, \theta, \eta, \phi, \lambda)^{T}$ be the parameter vector. The log-likelihood function of (2.1) is given by

$$
\begin{aligned}
\log L= & n \log \phi+\sum_{i=1}^{n} \log \left(\alpha+\eta \theta x_{i}^{\theta-1}\right)-\alpha \sum_{i=1}^{n} x_{i}-\eta \sum_{i=1}^{n} x_{i}^{\theta}+(\phi-1) \sum_{i=1}^{n} \log \left\{1-\exp \left(-\alpha x_{i}-\eta x_{i}^{\theta}\right)\right\} \\
& +\sum_{i=1}^{n} \log \left\{1+\lambda-2 \lambda\left[1-\exp \left\{-\alpha x_{i}-\eta x_{i}^{\theta}\right\}\right]^{\phi}\right\} .
\end{aligned}
$$


The components of the score function can be obtained by differentiating (6.1) with respect to $\alpha, \theta, \eta, \phi$ and $\lambda$, then equating it to zero, we obtain

$$
\begin{aligned}
\frac{\partial \log L}{\partial \alpha}= & \sum_{i=1}^{n}\left(\alpha+\eta \theta x_{i}^{\theta-1}\right)^{-1}+(\phi-1) \sum_{i=1}^{n} \frac{x_{i} \exp \left(-\alpha x_{i}-\eta x_{i}^{\theta}\right)}{\left\{1-\exp \left(-\alpha x_{i}-\eta x_{i}^{\theta}\right)\right\}} \\
& -\sum_{i=1}^{n} x_{i}-\sum_{i=1}^{n} \frac{2 \lambda \phi x_{i}\left[1-\exp \left\{-\alpha x_{i}-\eta x_{i}^{\theta}\right\}\right]^{\phi-1} \exp \left\{-\alpha x_{i}-\eta x_{i}^{\theta}\right\}}{\left\{1+\lambda-2 \lambda\left[1-\exp \left\{-\alpha x_{i}-\eta x_{i}^{\theta}\right\}\right]^{\phi}\right\}} \\
\frac{\partial \log L}{\partial \theta}= & \sum_{i=1}^{n} \eta\left(\alpha+\eta \theta x_{i}^{\theta-1}\right)^{-1} x_{i}^{\theta-1}\left(\frac{\theta(\theta-1)}{x_{i}}+1\right)+\eta(\phi-1) \sum_{i=1}^{n} \frac{\exp \left(-\alpha x_{i}-\eta x_{i}^{\theta}\right) x_{i}^{\theta} \log x_{i}}{\left\{1-\exp \left(-\alpha x_{i}-\eta x_{i}^{\theta}\right)\right\}} \\
& -\eta \sum_{i=1}^{n} x_{i}^{\theta} \log x_{i}-\sum_{i=1}^{n} \frac{2 \lambda \eta \phi\left[1-\exp \left\{-\alpha x_{i}-\eta x_{i}^{\theta}\right\}\right]^{\phi-1} \exp \left\{-\alpha x_{i}-\eta x_{i}^{\theta}\right\} x_{i}^{\theta} \log x_{i}}{\left\{1+\lambda-2 \lambda\left[1-\exp \left\{-\alpha x_{i}-\eta x_{i}^{\theta}\right\}\right]^{\phi}\right\}} \\
\frac{\partial \log L}{\partial \eta}= & \sum_{i=1}^{n} \theta x_{i}^{\theta-1}\left(\alpha+\eta \theta x_{i}^{\theta-1}\right)^{-1}+(\phi-1) \sum_{i=1}^{n} \frac{x_{i}^{\theta} \exp \left(-\alpha x_{i}-\eta x_{i}^{\theta}\right)}{\left\{1-\exp \left(-\alpha x_{i}-\eta x_{i}^{\theta}\right)\right\}} \\
& -\sum_{i=1}^{n} x_{i}^{\theta}-\sum_{i=1}^{n} \frac{2 \lambda \phi x_{i}^{\theta}\left[1-\exp \left\{-\alpha x_{i}-\eta x_{i}^{\theta}\right\}\right]^{\phi-1} \exp \left\{-\alpha x_{i}-\eta x_{i}^{\theta}\right\}}{\left\{1+\lambda-2 \lambda\left[1-\exp \left\{-\alpha x_{i}-\eta x_{i}^{\theta}\right\}\right]^{\phi}\right\}} \\
\frac{\partial \log L}{\partial \phi}= & \frac{n}{\phi}+\sum_{i=1}^{n}\left\{1-\exp \left(-\alpha x_{i}-\eta x_{i}^{\theta}\right)\right\}-2 \lambda \sum_{i=1}^{n} \frac{\left[1-\exp \left\{-\alpha x_{i}-\eta x_{i}^{\theta}\right\}\right]^{\phi} \log \left[1-\exp \left\{-\alpha x_{i}-\eta x_{i}^{\theta}\right\}\right]}{\left\{1+\lambda-2 \lambda\left[1-\exp \left\{-\alpha x_{i}-\eta x_{i}^{\theta}\right\}\right]^{\phi}\right\}}
\end{aligned}
$$

and

$$
\frac{\partial \log L}{\partial \lambda}=\sum_{i=1}^{n} \frac{1-2\left[1-\exp \left\{-\alpha x_{i}-\eta x_{i}^{\theta}\right\}\right]^{\phi}}{\left\{1+\lambda-2 \lambda\left[1-\exp \left\{-\alpha x_{i}-\eta x_{i}^{\theta}\right\}\right]^{\phi}\right\}}
$$

respectively. We obtain the analytical expressions for the MLEs of a five-parameter score vector, yields the ML estimators $\hat{\alpha}, \hat{\theta}, \hat{\eta}, \hat{\phi}$ and $\hat{\lambda}$ of the TNGW distribution. These parameters can be estimated by using the BFGS method in R package "Adequacy Model" (http://www.r-project.org). We required the observed information matrix for the interval estimation and hypothesis testing. All the second order derivatives exist for the five-parameter TNGW distribution. Thus we have the observed information matrix as

$$
(\hat{\alpha}, \hat{\theta}, \hat{\eta}, \hat{\phi}, \hat{\lambda})^{T} \sim N_{5}\left\{(\alpha, \theta, \eta, \phi, \lambda)^{T}, K(\Theta)^{-1}\right\}
$$

where $K(\Theta)^{-1}$ is the variance covariance matrix of the unknown parameters having the components $K_{\vartheta_{i} \vartheta_{j}}=\partial^{2} \log L / \partial \vartheta_{i} \vartheta_{j}, i, j=1,2,3,4,5$. The asymptotic multivariate normal $N_{5}\left\{0, K(\Theta)^{-1}\right\}$ distribution can be used to construct the confidence intervals for each parameters $\vartheta$. An approximate $100(1-\gamma) \%$ asymptotic confidence intervals (ACI) for each parameter $\alpha, \theta, \eta, \phi$ and $\lambda$ can be deter- 


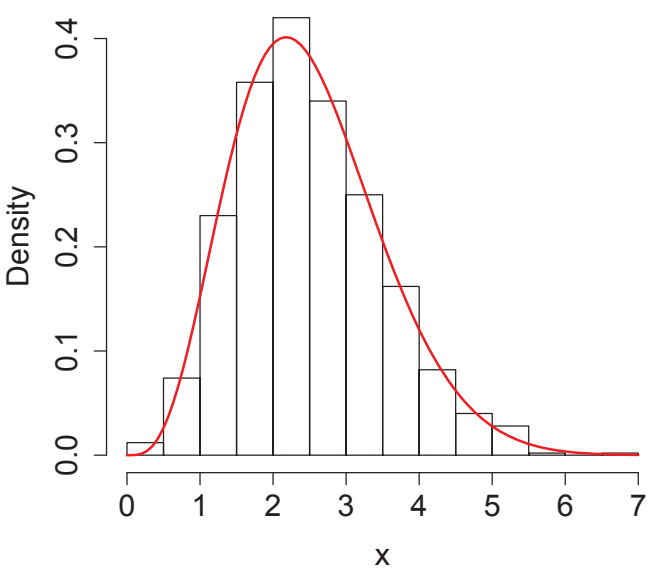

(a) $\alpha=1 ; \phi=3 ; \eta=2 ; \theta=2 ; \lambda=0.5$

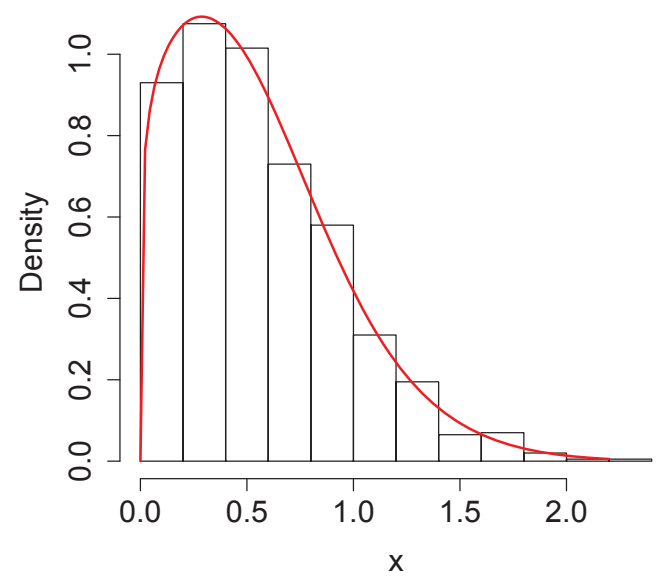

(b) $\alpha=0.5 ; \phi=1 ; \eta=2 ; \theta=1 ; \lambda=0.5$

Figure 5: Plots of the TNGW densities for simulated data sets.

mined as

$$
\mathrm{ACI}_{r}=\left(\hat{\vartheta}_{r}-Z_{\frac{\gamma}{2}} \sqrt{-k_{r, r}}, \hat{\vartheta}_{r}+Z_{\frac{\gamma}{2}} \sqrt{-k_{r, r}}\right),
$$

where $-k_{r, r}$ represents the elements of the observed information matrix and $Z_{\gamma / 2}$ is the upper $\gamma^{t h}$ percentile of the standard normal distribution.

We conducted the simulation study to evaluate the performance of MLEs with respect to the sample size for the TNGW distribution. By using equation (3.1), we generated samples from the TNGW distribution for different sizes $n=50,100,200,400,800$ for the fixed choice of the parameters $\alpha=0.5, \theta=2, \eta=1, \phi=0.5$ and $\lambda=0.5$. In the context of the computational complexities, the quantile model involves non-linear equation and needs to be calculated by some iterative process. The simulation process is repeated 500 times using the BFGS optimization method in R. We fitted the TNGW distribution for these samples using the maximum likelihood method. Table 6 describes the results for five different parameter values $\alpha, \theta, \eta, \phi, \lambda$ with their corresponding standard errors, bias and mean square error (MSE). Table 4 reports that the simulated results are quite promising with respect to the increasing sample size. The histogram of two simulated data sets for some selected values of parameters based on 1,000 observations are displayed in Figure 5.

\section{Application}

This section illustrates the usefulness of the TNGW distribution with nicotine cigarette data. The data set consists of 396 observations of nicotine content in milligrams in cigarettes for several cigarettes brands in 1995. The data was obtained from the Federal Trade Commission (FTC), an independent agency of the US government, whose main mission is the promotion of consumer protection. The report entitled "Tar, Nicotine and Carbon Monoxide of the Smoke of 1249 varieties of domestic cigarettes for the Year 1995" at FTC (1998) consists of data sets and information about the source of data, smoker behavior and beliefs about nicotine, tar and carbon monoxide contents in cigarettes. The model parameters are estimated using the method of maximum likelihood and five goodness-of-fit statistics are used to compare TNGW distribution with other five lifetime models, their associated 
Table 6: Monte Carlo simulation results based on MLE, S.E, Bias and MSE for the TNGW distribution

\begin{tabular}{|c|c|c|c|c|c|}
\hline$n$ & Parameter & Mean & S.E & Bias & MSE \\
\hline \multirow{5}{*}{50} & $\alpha$ & 0.1009 & 0.7272 & -0.3991 & 0.6881 \\
\hline & $\theta$ & 1.6416 & 0.8418 & -0.3584 & 0.8371 \\
\hline & $\eta$ & 1.5098 & 0.9281 & 0.5098 & 1.1213 \\
\hline & $\phi$ & 0.4551 & 0.3787 & -0.0449 & 0.1454 \\
\hline & $\lambda$ & 0.4438 & 0.6967 & -0.0562 & 0.4885 \\
\hline \multirow{5}{*}{100} & $\alpha$ & 0.6365 & 0.8028 & 0.1365 & 0.6631 \\
\hline & $\theta$ & 1.7085 & 0.5387 & -0.2915 & 0.3752 \\
\hline & $\eta$ & 0.7689 & 0.7212 & -0.2311 & 0.5735 \\
\hline & $\phi$ & 0.6048 & 0.1507 & 0.1048 & 0.0337 \\
\hline & $\lambda$ & 0.7595 & 0.3492 & 0.2595 & 0.1893 \\
\hline \multirow{5}{*}{200} & $\alpha$ & 0.6783 & 0.8762 & 0.1783 & 0.7995 \\
\hline & $\theta$ & 1.7260 & 0.5125 & -0.2740 & 0.3377 \\
\hline & $\eta$ & 1.3868 & 1.4812 & 0.3868 & 2.3435 \\
\hline & $\phi$ & 0.4933 & 0.0795 & -0.0067 & 0.0064 \\
\hline & $\lambda$ & 0.0113 & 0.7808 & -0.4887 & 0.8485 \\
\hline \multirow{5}{*}{400} & $\alpha$ & 0.4748 & 0.2600 & -0.0252 & 0.0682 \\
\hline & $\theta$ & 2.1514 & 0.2564 & 0.1514 & 0.0886 \\
\hline & $\eta$ & 0.9169 & 0.2982 & -0.0831 & 0.0958 \\
\hline & $\phi$ & 0.5194 & 0.0552 & 0.0194 & 0.0034 \\
\hline & $\lambda$ & 0.6142 & 0.2851 & 0.1142 & 0.0943 \\
\hline \multirow{5}{*}{800} & $\alpha$ & 0.5961 & 0.2093 & 0.0961 & 0.0530 \\
\hline & $\theta$ & 2.3019 & 0.2544 & 0.3019 & 0.1558 \\
\hline & $\eta$ & 0.8400 & 0.2618 & -0.1600 & 0.0941 \\
\hline & $\phi$ & 0.5490 & 0.0383 & 0.0490 & 0.0038 \\
\hline & $\lambda$ & 0.6526 & 0.2559 & 0.1526 & 0.0887 \\
\hline
\end{tabular}

MLE = maximum likelihood estimate; MSE = mean square error.

density functions are given by

1. Kumaraswamy Weibull Poisson (KwWP) distribution with pdf

$$
\begin{aligned}
f(x)= & a b c \lambda \beta^{c} x^{c-1}\left[1-\exp \left(-(x \beta)^{c}\right)\right]^{a-1}\left[1-\left(1-\exp \left(-(\beta x)^{c}\right)\right)^{a}\right]^{b-1} \\
& \times \frac{\exp \left[-\lambda\left\{1-\left(1-\left(1-\exp \left(-(\beta x)^{c}\right)\right)^{a}\right)^{b}\right\}-(\beta x)^{c}\right]}{(1-\exp (-\lambda))}
\end{aligned}
$$

where $a, b, c>0$ are the shape parameters and $\beta, \lambda>0$ are the scale parameters of the KwWP distribution proposed by Ramos et al. (2015).

2. Transmuted additive Weibull (TAW) distribution with pdf

$$
f(x)=\left(\alpha \theta x^{\theta-1}+\eta \beta x^{\beta-1}\right) \exp \left(-\alpha x^{\theta}-\eta x^{\beta}\right)\left\{1-\lambda+2 \lambda \exp \left(-\alpha x^{\theta}-\eta x^{\beta}\right)\right\},
$$

where $\alpha, \eta>0$ are the scale parameters, $\beta, \theta>0$ are the shape parameters and $\lambda$ is the transmuted parameter of the TAW distribution proposed by Elbatal and Aryal (2013).

3. New generalized Weibull (NGW) distribution with pdf

$$
f(x)=\phi\left(\alpha+\eta \theta x^{\theta-1}\right) \exp \left\{-\alpha x-\eta x^{\theta}\right\}\left[1-\exp \left\{-\alpha x-\eta x^{\theta}\right\}\right]^{\phi-1},
$$

where $\alpha, \eta>0$ are the scale parameters and $\theta, \phi>0$ are the shape parameter of the NGW distribution proposed by Zaindin and Sarhan (2011). 
Table 7: Estimates of the model parameters for the nicotine in cigarettes data

\begin{tabular}{|c|c|c|c|c|c|}
\hline Distribution & & & Estimates & & \\
\hline $\operatorname{GPW}(\alpha, \beta)$ & $\begin{array}{c}2.6135 \\
(0.1054)\end{array}$ & $\begin{array}{c}1.3514 \\
(0.0422)\end{array}$ & & & \\
\hline $\operatorname{EW}(\phi, \eta, \theta)$ & $\begin{array}{c}0.8851 \\
(0.1742)\end{array}$ & $\begin{array}{c}1.4647 \\
(0.2082)\end{array}$ & $\begin{array}{c}3.0386 \\
(0.3637)\end{array}$ & & \\
\hline $\operatorname{NGW}(\phi, \alpha, \eta, \theta)$ & $\begin{array}{c}1.6403 \\
(0.5489)\end{array}$ & $\begin{array}{c}0.4030 \\
(0.4101)\end{array}$ & $\begin{array}{c}1.6472 \\
(0.1628)\end{array}$ & $\begin{array}{c}2.7252 \\
(0.2035)\end{array}$ & \\
\hline $\operatorname{TAW}(\alpha, \beta, \eta, \theta, \lambda)$ & $\begin{array}{c}1.4979 \\
(0.1349)\end{array}$ & $\begin{array}{c}1.1698 \\
(0.6121)\end{array}$ & $\begin{array}{c}0.0762 \\
(0.1016)\end{array}$ & $\begin{array}{c}3.0361 \\
(0.1868)\end{array}$ & $\begin{array}{c}0.0113 \\
(0.0252)\end{array}$ \\
\hline $\operatorname{KwWP}(a, b, c, \lambda, \beta)$ & $\begin{array}{c}0.7905 \\
(0.1439)\end{array}$ & $\begin{array}{c}0.2064 \\
(0.0283)\end{array}$ & $\begin{array}{c}3.0436 \\
(0.0228)\end{array}$ & $\begin{array}{c}0.0215 \\
(0.4799)\end{array}$ & $\begin{array}{c}1.9227 \\
(0.0396)\end{array}$ \\
\hline $\operatorname{TNGW}(\phi, \alpha, \eta, \theta, \lambda)$ & $\begin{array}{c}1.5793 \\
(0.5532)\end{array}$ & $\begin{array}{c}0.3098 \\
(0.3432)\end{array}$ & $\begin{array}{c}1.2887 \\
(0.2270)\end{array}$ & $\begin{array}{c}2.9588 \\
(0.2259)\end{array}$ & $\begin{array}{c}0.4920 \\
(0.2617)\end{array}$ \\
\hline
\end{tabular}

Table 8: Goodness-of-fit statistics for the nicotine in cigarettes data

\begin{tabular}{lccccc}
\hline \hline \multicolumn{1}{c}{ Distribution } & AIC & CAIC & $A^{*}$ & $W^{*}$ & K-S test [ $p$-value] \\
\hline GPW $(\alpha, \beta)$ & 142.48 & 142.51 & 4.0559 & 0.7726 & $0.1243[9.54 \mathrm{E}-6]$ \\
$\operatorname{EW}(\phi, \eta, \theta)$ & 143.70 & 143.76 & 3.9705 & 0.7510 & $0.1194[2.47 \mathrm{E}-5]$ \\
$\operatorname{NGW}(\phi, \alpha, \eta, \theta)$ & 140.39 & 140.49 & 3.6479 & 0.6904 & $0.1213[1.71 \mathrm{E}-5]$ \\
$\operatorname{TAW}(\alpha, \beta, \eta, \theta, \lambda)$ & 144.38 & 144.53 & 3.8011 & 0.7244 & $0.1249[8.50 \mathrm{E}-6]$ \\
$\operatorname{KWWP}(a, b, c, \lambda, \beta)$ & 146.56 & 146.71 & 3.9465 & 0.7538 & $0.1250[8.43 \mathrm{E}-6]$ \\
$\operatorname{TNGW}(\phi, \alpha, \eta, \theta, \lambda)$ & 140.36 & 140.47 & 3.4984 & 0.6577 & $0.1176[3.45 \mathrm{E}-5]$ \\
\hline \hline
\end{tabular}

$\mathrm{AIC}=$ Akaike information criterion, CAIC = consistent Akaike information criterion, $A^{*}=$ Anderson-Darling,

$W^{*}=$ Cramér-von Mises.

4. Exponentiated Weibull (EW) distribution with pdf

$$
f(x)=\phi \eta \theta x^{\theta-1} \exp \left(-\eta x^{\theta}\right)\left\{1-\exp \left(-\eta x^{\theta}\right)\right\}^{\phi-1},
$$

where $\eta>0$ is the scale parameter and $\phi, \theta>0$ are the shape parameters of the EW distribution introduced by Mudholkar and Srivastava (1993).

5. Generalized power Weibull (GPW) distribution with pdf

$$
f(x)=\alpha \beta x^{\alpha-1}\left(1+x^{\alpha}\right)^{\beta-1} \exp \left(1-\left(1+x^{\alpha}\right)^{\beta}\right),
$$

where $\alpha, \beta>0$ are the shape parameters of the GPW distribution proposed by Nikulin and Haghighi (2006).

The required numerical evaluations are implemented using $\mathrm{R}$ language. The MLEs of the parameters (with their standard errors) for the nicotine in cigarettes data are displayed in Table 7. Table 8 illustrates their corresponding values of the Akaike Information Criterion (AIC), Consistent Akaike Information Criterion (CAIC), Anderson-Darling $\left(A^{*}\right)$, the Cramér-von Mises $\left(W^{*}\right)$ and the K-S test goodness-of-fit statistics to verify which model fits better. The results suggest that the TNGW distribution has the smallest values of these statistics; therefore, the TNGW model can be chosen as the best model among the six fitted models. Figure 6 shows the density functions with histogram and empirical fitted plots of the six distributions. From the visualization of density functions and Table 8 indicate that the TNGW distribution provides a better fit than the other five distributions. Therefore, the TNGW distribution can be chosen as the best model for nicotine in cigarettes data in terms of model fitting. The estimated fitted survival function and P-P plot of the TNGW model for the nicotine 


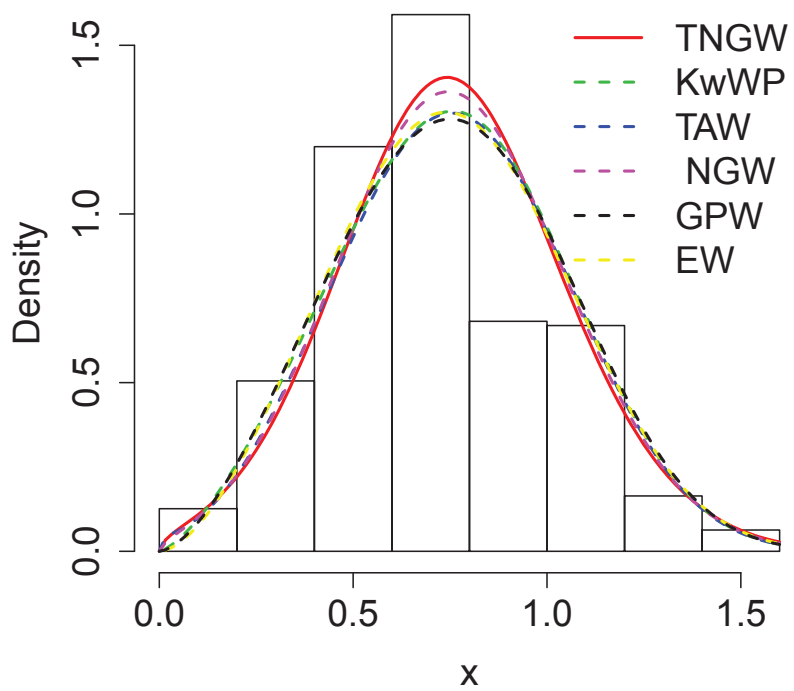

Figure 6: Estimated densities of the TNGW, KWWP, TAW, NGW, GPW and EW models for the nicotine in cigarettes data.
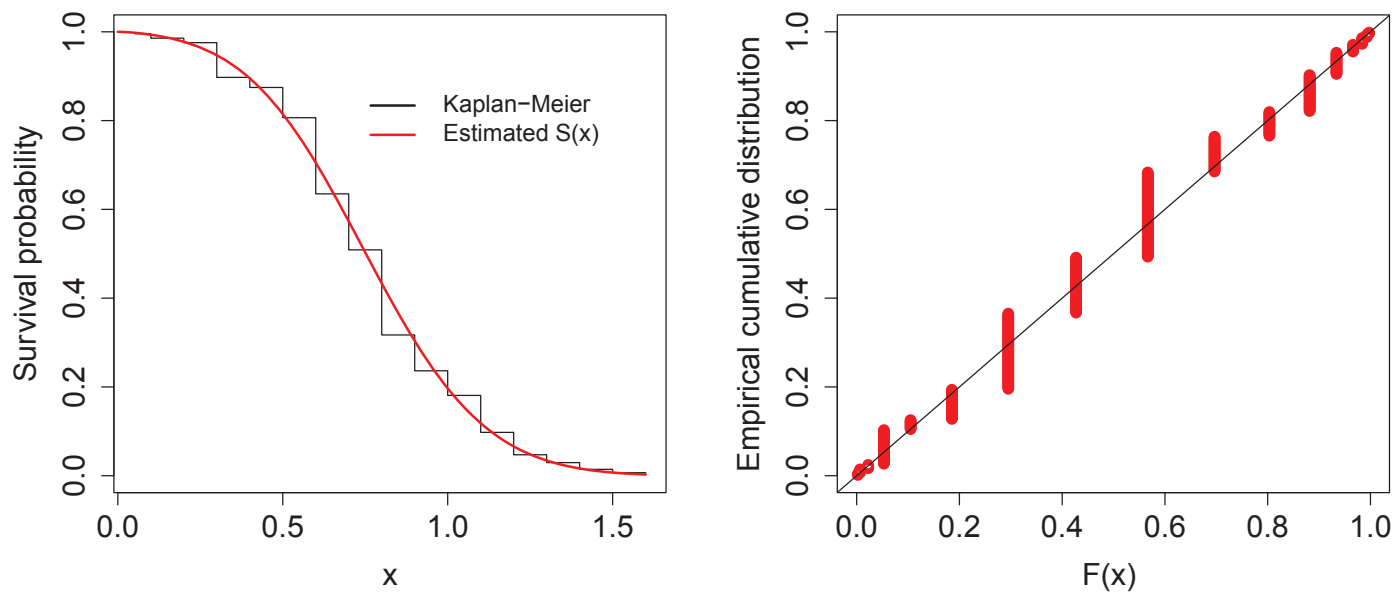

Figure 7: Estimated fitted survival function and P-P plot of the TNGW model for the nicotine in cigarettes data.

in cigarettes data are displayed in Figure 7. These plots suggest that the TNGW distribution could be chosen as the best model because it does fit better to the P-P plot and survival curve for the nicotine in cigarettes data; therefore, we conclude that the TNGW model has better relationship for the nicotine in cigarettes data.

\section{Conclusion}

In this paper, we have constructed the five-parameter distribution (referred to as the TNGW distribution), which includes twenty-three lifetime distributions as special cases, as well as study its theo- 
retical properties. The TNGW model is more flexible than the KwWP, TAW, NGW, EW, and GPW distributions proposed recently in the literature. We obtained the analytical shapes of density and hazard functions of the TNGW distribution. The TNGW distribution has increasing, decreasing and constant failure rate patterns for lifetime data. The flexibility and usefulness of the TNGW model is illustrated in an application to nicotine in cigarettes data using MLE.

\section{References}

Abernethy RB (2000). The New Weibull Handbook (4th ed), R. B. Abernethy, North Palm Beach, FL. Aryal GR and Tsokos CP (2011). Transmuted Weibull distribution: a generalization of the Weibull probability distribution, European Journal of Pure and Applied Mathematics, 4, 89-102.

Ashour SK and Eltehiwy MA (2013). Transmuted exponentiated modified Weibull distribution, International Journal of Basic and Applied Sciences, 2, 258-269.

Bourguignon M, Ghosh I, and Cordeiro GM (2016). General results for the transmuted family of distributions and new models, Journal of Probability and Statistics, 2016, Article ID 7208425. http://dx.doi.org/10.1155/2016/7208425

Cordeiro GM, Gomes AE, da-Silva CQ, and Ortega EMM (2013). The beta exponentiated Weibull distribution, Journal of Statistical Computation and Simulation, 83, 114-138.

Cordeiro GM, Ortega EMM, and Silva GO (2014). The Kumaraswamy modified Weibull distribution: theory and applications, Journal of Statistical Computation and Simulation, 84, 1387-1411.

Elbatal I and Aryal G (2013). On the transmuted additive Weibull distribution, Austrian Journal of Statistics, 42, 117-132.

Federal Trade Commission (1998). Results of tar, nicotine and carbon monoxide testing for 1,249 varieties of domestic cigarettes sold in 1995, FTC File No. 962 3099, Retrieved September 19, 2016, from: https://www.ftc.gov/news-events/press-releases/1998/01/results-tar-nicotine-and-ca rbon-monoxide-testing-1249-varieties

Havrda J and Charvát F (1967). Quantification method in classification processes: concept of structural $\alpha$-entropy, Kybernetika, 3, 30-35.

Khan MS and King R (2013a). Transmuted modified Weibull distribution: a generalization of the modified Weibull probability distribution, European Journal of Pure and Applied Mathematics, 6, 66-88.

Khan MS and King R (2013b). Transmuted generalized inverse Weibull distribution, Journal of Applied Statistical Sciences, 20, 15-32.

Khan MS and King R (2014). A new class of transmuted inverse Weibull distribution for reliability analysis, American Journal of Mathematical and Management Sciences, 33, 261-286.

Khan MS and King R (2015). Transmuted modified inverse Rayleigh distribution, Austrian Journal of Statistics, 44, 17-29.

Khan MS, King R, and Hudson I (2014). Characterizations of the transmuted inverse Weibull distribution, ANZIAM Journal, 55, C197-C217.

Merovci F (2013). Transmuted Rayleigh distribution, Austrian Journal of Statistics, 42, 21-31.

Mudholkar GS and Srivastava DK (1993). Exponentiated Weibull family for analyzing bathtub failure data, IEEE Transactions on Reliability, 42, 299-302.

Nadarajah S and Kotz S (2006). The exponentiated type distributions, Acta Applicandae Mathematica, 92, 97-111.

Nikulin M and Haghighi F (2006). A chi-squared test for the generalized power Weibull family for the head-and-neck cancer censored data, Journal of Mathematical Sciences, 133, 1333-1341. 
Ramos MWA, Marinho PRD, Cordeiro GM, da Silva RV, and Hamedani G (2015). The Kumaraswamy -G Poisson family of distributions, Journal of Statistical Theory and Applications, 14, 222-239.

Rényi, A. (1961). On measures of information and entropy, In Proceedings of the fourth Berkeley Symposium on Mathematics, Statistics and Probability, Berkeley, CA, 547-561.

Sarhan AM and Zaindin M (2009). Modified Weibull distribution, Applied Sciences, 11, 123-136.

Sharma VK, Singh SK, and Singh U (2014). A new upside-down bathtub shaped hazard rate model for survival data analysis, Applied Mathematics and Computation, 239, 242-253.

Shaw WT and Buckley IR (2009). The alchemy of probability distributions: beyond Gram-Charlier expansions, and a skew-kurtotic normal distribution from a rank transmutation map, Retrieved September 19, 2016, from: http://arxiv.org/abs/0901.0434

Silva GO, Ortega EMM, and Cordeiro GM (2010). The beta Modified Weibull distribution, Lifetime Data Analysis, 16, 409-430.

Tian Y, Tian M, and Zhu Q (2014). Transmuted linear exponential distribution: a new generalization of the linear exponential distribution, Communications in Statistics-Simulation and Computation, 43, 2661-2677.

Xie M and Lai CD (1996). Reliability analysis using an additive Weibull model with bathtub-shaped failure rate function, Reliability Engineering \& System Safety, 52, 87-93.

Xie M, Tang Y, and Goh TN (2002). A modified Weibull extension with bathtub-shaped failure rate function, Reliability Engineering \& System Safety, 76, 279-285.

Zaindin M and Sarhan AM (2011). New generalized Weibull distribution, Pakistan Journal of Statistics, 27, 13-30. 